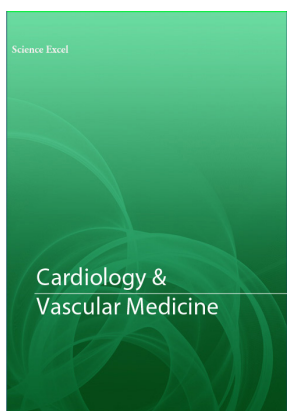

Correspondence

Dr. Victor Gurewich, MD

Director Vascular Research Laboratory, Mount Auburn Hospital, Cambridge, USA.

- Received Date: 24 May 2020;

- Accepted Date: 09 June 2020;

- Publication Date: 15 June 2020.

Copyright

(c) 2020 Science Excel. This is an openaccess article distributed under the terms of the Creative Commons Attribution 4.0 International license.

\title{
The Assumption That Fibrinolysis and tPA Can Be Equated is Mistaken
}

\author{
Dr. Victor Gurewich, MD \\ Director Vascular Research Laboratory, Mount Auburn Hospital, Cambridge, USA. \\ Professor of Medicine, Harvard Medical School, USA.
}

\section{Case Summary}

A 57-year-old gentleman was referred by GP to our Acute Fibrinolysis is the body's defense system against obstructive blood clots, which are responsible for conditions like heart attack and stroke. This important endogenous function is mediated not by one, but by two activators called tissue plasminogen activator (tPA) and urokinase plasminogen activator (uPA). Since tPA was approved in 1987 and it binds to the fibrin clot and induces fibrin-specific fibrinolysis. By contrast, uPA was known since non-specifc plasminogen activator which has no fibrin affinity. Not surprisingly, tPA has invariably dominated fibrinolytic therapy.

For example, in review articles dealing with fibrinolysis, it is taken for granted that tPA is the activator responsible so that it is often not even mentioned. This is a well established concept and a Google search of publications in which Fibrinolysis is in the title, lists more than 350,000 citations in which tPA is assumed to be the activator.

At the same time, two independent gene deletion studies showed that, when the tPA gene was deleted in animals, little change in the animals' fibrinolytic effect took place. Since, when the uPA gene was deleted, significant inhibition of fibrinolysis occurred. When both of the activator genes were deleted, fibrinolysis was arrested, and extensive fibrin deposition was found in the animals. It was concluded that both activators were needed for normal function but that UPA rather than tPA was the dominant activator $[1,2]$, contrary to the prevailing belief. However, these findings had little influence on the prevailing idea that tPA was responsible for fibrinolysis.

In standard fibrinolytic therapy, the tPA concentration in blood is almost a thousand-fold higher the normal, physiological concentration of about $5 \mathrm{ng} / \mathrm{ml}$. And yet, therapeutic and physiological fibrinolytic efficacies are not similarly disparate. For example, in fibrinolytic therapy of heart attack, in which $100 \mathrm{mg}$ of tPA is infused, complete patency of the responsible coronary artery at 24 hours was seen in $45 \%$ of patients [3]. By comparison, in 15\% of untreated heart attack patients, the infarct artery was already patent at the time of the initial therapeutic catheterization, representing physiological fibrinolysis [4].

Therefore, physiological fibrinolysis, with only about a thousandth of the tPA concentration, was only 3 -fold less effective than therapeutic fibrinolysis. This relative efficacy of physiological fibrinolysis suggests that more than tPA must have been involved.

\section{Fibrinolysis requires both activators}

The other biological plasminogen activator is urokinase plasminogen activator (uPA) in the blood. The native form of uPA is a single-chain proenzyme, prourokinase (proUK), first discovered in 1981 [5]. Prior to this time the only known form of uPA was a two-chain, non-specific enzyme which was believed to be only an extravascular activator, due to a UPA receptor (UPAR) present on many cells by which extravascular functions like cell migration take place.

ProUK is a generally unrecognized component of the intravascular fibrinolytic system, that is a proenzyme in blood, whereas tPA is an enzyme stored in the vascular endothelium. When an intravascular thrombus forms, tPA is released locally and binds to the thrombus. Binding is due to tPA's high fibrin affinity, a property unique to tPA and has been used to isolate it directly from plasma [5]. The tPA binding site on the fibrin clot is proximal to a fibrin-bound plasminogen [6], forming a ternary complex on the fibrin surface that promotes tPA's activation of plasminogen 1,000-fold and initiates fibrin degradation [7].

Since tPA has no second comparable fibrin binding site and in the absence of the ternary complex promotion, tPA is a weak plasminogen activator [8], tPA's fibrinolytic function is limited to this step. The initiation of fibrin degradation by tPA creates two additional plasminogen binding sites [9], and these plasminogens are activated by uPA. The first one is activated by proUK [10] that is then reciprocally activated by plasmin to $\mathrm{UK}$, which activates the remaining plasminogen, completing fibrinolysis [9]. This sequence explains why both activators are required $\mathrm{n}$ fibrinolysis as also evidenced by the gene deletion studies $[1,2]$. 
Therefore, fibrinolysis involves the sequential activation of three fibrin-bound plasminogens [11], where tPA activates the first and uPA the remaining two. This physiological mechanism is analogous to an automobile engine, where tPA is the starter and uPA the fuel. As with the automobile, neither component can function effectively without the other, and although their functions are independent, they are interdependent.

In view of this mechanism, it is not surprising that standard monotherapy with tPA alone has fallen short and is inefficient, requiring very high doses that are associated with bleeding complications. Since tPA has high fibrin affinity, when it is administered by a lengthy infusion, it puts hemostatic fibrin at risk and disruption of hemostatic is the main cause of tPA bleeding complications.

By contrast, when is administered as a bolus, for which tPA's high fibrin affinity is well adapted, much smaller, safer doses are needed. This was verified in a clinical trial of heart attack. In the PATENT trial [12], 101 patients with heart attack were given a $5 \mathrm{mg}$ bolus of tPA which was followed by a 90 -minute infusion of proUK at a rate of $40 \mathrm{mg} / \mathrm{h}$. An $82 \%$ complete patency of the coronary artery at 24 $\mathrm{h}$ was obtained, and the 30 -day mortality was $1 \%$. These results are far better than those of GUSTO, the best of the tPA mega trials, in which the $24 \mathrm{~h}$ coronary patency was only $45 \%$ and the mortality was $6.3 \%$ [3]. These findings confirm the dual activator requirement for effective fibrinolysis.

\section{Conclusion}

The prevailing idea that fibrinolysis is due to the activity of tPA alone has been a mistake that still remains to be corrected. Standard fibrinolytic therapy has not changed since 1987 when tPA was first approved. Since tPA never lived up to expectations, fibrinolysis was discredited and replaced by a more timing consuming and costly hospital procedure and percutaneous intervention has become the treatment of choice. Nevertheless, the simplest and fastest method to restore perfusion of a thrombus occluded artery remains fibrinolysis. The loss of interest in this valuable treatment modality has interfered with improving it, since fibrinolysis was abandoned before it was fully understood. This short review has tried to explain why this is the case.

\section{References}

1. Bugge TH, Flick MJ, Danton MJS, et al. Urokinase-type plasminogen activator is effective in fibrin clearance in the absence of its receptor or tissue-type plasminogen activator.
Proc Natl Acad Sci USA 1996; 93:5899-5904.

2. Singh I, Burnand KG, Collins M, et al. Failure of thrombus to resolve in urokinase-type plasminogen activator gene-knockout mice: rescue by normal bone marrow-derived cells. Circulation 2003;107:869-875.

3. GUSTO Angiographic Investigators. The effects of tissue plasminogen activator, streptokinase, or both on coronaryartery patency, ventricular function, and survival after acute myocardial infarction. N Engl J Med 1993;329:1615-1622.

4. Assessment of the Safety and Efficacy of a New Treatment Strategy with Percutaneous Coronary Intervention (ASSENT-4 PCI) Investigators. Primary versus tenecteplase-facilitated percutaneous coronary intervention in patients with STsegment elevation acute myocardial infarction (ASSENT-4 PCI): randomized trial. Lancet 2006;367:569-578.

5. Husain SS, Lipinski B, Gurewich V. Rapid purification of high affinity plasminogen activator from human plasma by specific adsorption on fibrin-celite. Proc Nat Acad Sci (USA) 1981;78:4265-4269.

6. Voskuilen M, Vermond A, Veeneman GH, et al. Fibrinogen lysine residue Aa157plays a crucial role in the fibrin-induced acceleration of plasminogen activation, catalyzed by tissue-type plasminogen activator. J Biol Chem 1987;262:5944-5946.

7. Hoylaerts M, Rijken DC, Lijnen $H R$, et al.Kinetics of the activation of plasminogen by human tissue plasminogen activator. Role of fibrin. J Biol Chem 1982;257:2912-2919.

8. Collen D. The plasminogen (fibrinolytic) system. Thromb Haemost 1999;82:259-270.

9. Petersen LC. Kinetics of reciprocal prourokinase/plasminogen activation. Stimulation by a template formed by the urokinase receptor bound to poly (D-lysine). Eur J Biochem 1997;245:316323.

10. Liu J, Gurewich V. Fragment E-2 from fibrin substantially enhances pro-urokinase-induced glu-plasminogen activation. A kinetic study using a plasmin-resistant mutant pro-urokinase (Ala-158-rpro-UK). Biochemistry 1992;31:6311-6317.

11. Suenson E, Lützen $O$, Thorsen S. Initial plasmin-degradation of fibrin as the basis of a positive feed-back mechanism in fibrinolysis. Eur J Biochem 1984;140:513-522.

12. Zarich SW, Kowalchuk GJ, Weaver WD, et al. Sequential combination thrombolytic therapy for acute myocardial infarction: results of the pro-urokinase and t-PA enhancement of thrombolysis (PATENT) trial. J Am Coll Cardio 1995;26:374379. 